\title{
Utilization of Genetic Algorithm in Allocating Goods to Shop Shelves Under an Application to Cup Noodles
}

\author{
Yuki Higuchi ${ }^{1}$, Koumei Suzuki ${ }^{2} \&$ Kazuhiro Takeyasu ${ }^{3}$ \\ ${ }^{1}$ Faculty of Business Administration, Setsunan University, Japan \\ ${ }^{2}$ Only Sky Co., Shizuoka, Japan \\ ${ }^{3}$ College of Business Administration, Tokoha University, Japan \\ Correspondence: Yuki Higuchi, Faculty of Business Administration, Setsunan University, Japan.
}

Received: April 3, 2019

Accepted: April 25, 2019

Online Published: April 29, 2019

doi:10.5430/ijba.v10n3p104

URL: https://doi.org/10.5430/ijba.v10n3p104

\begin{abstract}
How to allocate goods in shop shelves makes great influence to sales amount. Searching best fit allocation of goods to shelves is a kind of combinatorial problem. This becomes a problem of integer programming and utilizing genetic algorithm may be an effective method. Reviewing past researches, there are few researches made on this. Formerly, we have presented papers concerning optimization in allocating goods to shop shelves utilizing genetic algorithm. In those papers, the problem that goods were not allowed to allocate in multiple shelves and the problem that goods were allowed to allocate in multiple shelves were pursued. In this paper, we examine the problem that does not allow goods to be allocated in multiple shelves and introduce the concept of sales profits and sales probabilities. Expansion of shelf is executed. Optimization in allocating goods to shop shelves is investigated. An application to the convenience store with POS sales data of cup noodles is executed. Utilizing genetic algorithm, optimum solution is pursued and verified by a numerical example. Comparison with other past papers was executed. Various patterns of problems must be examined hereafter.
\end{abstract}

Keywords: display, genetic algorithm, optimization, shelf

\section{Introduction}

Displaying method in the shop makes influence to sales amount, therefore various ideas are devised. What kind of items should be placed where in the shop, how to guide customers to what aisle in the shop are the big issues to be discussed. Searching best fit allocation of goods to shelves is also an important issue to be solved. In this paper, we seek how to optimize in allocating goods to shop shelves.

As for allocating good to shop shelves, following items are well known (Nagashima, 2005). Shelf height is classified as follows.

$>$ Shelf of $135 \mathrm{~cm}$ height: Customers can see the whole space of the shop. Specialty stores often use this type.

$>$ Shelf of $150 \mathrm{~cm}$ height: Female customers may feel pressure to the shelf height. This height may be the upper limit to look over the shop.

$>$ Shelf of $180 \mathrm{~cm}$ height: It becomes hard to look over the shop. Therefore it should not be used for island display (display at the center or inside the shop).

Next, we show the following three functions of shelf for display.

1. Exhibition of goods function

2. Stock function

3. Display function

Effective range for exhibition is generally said to be $45 \mathrm{~cm}-150 \mathrm{~cm}$. The range of $75 \mathrm{~cm}-135 \mathrm{~cm}$ is called golden zone especially. For the lower part under $45 \mathrm{~cm}$, goods are stocked as well as displaying.

Reviewing past papers, there are many papers concerning lay out problem. As for the problem of the distribution of equipment, we can see B. Korte et al. (2005), M. Gen et al. (1997) for the general research book. There are many 
researches made on this. Yamada et al. (2004) handles the lay out problem considering the aisle structure and intra-department material flow. Y. Wu et al. (2002) and Yamada et al. (2004) handle this problem considering aisle structure. Ito et al. (2006) considers multi-floor facility problem.

Although there are many researches on corresponding theme as stated above, we can hardly find researches on the problem of optimization in allocating goods to shop shelves.

Formerly, we have presented a paper concerning optimization in allocating goods to shop shelves utilizing genetic algorithm (Takeyasu et al.,2008) and many applications of them were presented (Shitara et al., 2015, Suzuki et al., 2015, Higuchi et al., 2016, Takeyasu et.al., 2016, Higuchi et al., 2017). In some of those papers, the problem that goods were not allowed to allocate in multiple shelves and the problem that goods were allowed to allocate in multiple shelves were pursued. In this paper, we examine the problem that does not allow goods to be allocated in multiple shelves and introduce the concept of sales profits and sales probabilities. Expansion of shelf is executed. Optimization in allocating goods to shop shelves is investigated. An application to the convenience store with POS sales data of cup noodles is executed. Utilizing genetic algorithm, optimum solution is pursued and verified by a numerical example. Comparison with other past papers was executed.

The rest of the paper is organized as follows. Problem description is stated in section 2. Genetic Algorithm is developed in section 3. Numerical example is exhibited in section 4 which is followed by the remarks of section 5 . Section 6 is a summary.

\section{Problem Description}

Shelf model is constructed as Figure 1. There are five shelf positions. Shelf position 1 is mainly to put big and heavy goods including stock function. Shelf position 3,4 at the height of the range $75 \mathrm{~cm}$ to $135 \mathrm{~cm}$ are the space of golden zone. Thus, we can use shelves properly by assuming these shelves. In numerical example, we examine using these five shelves. First of all, we make problem description in the case there is only one shelf (case 1). Then we expand to the case there are multiple shelves (case 2).

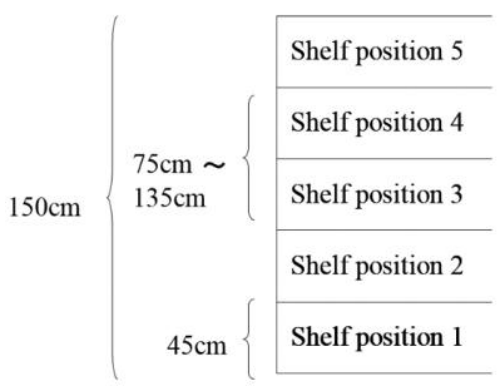

Figure 1. Shelf model

\subsection{Case 1: The Case That There Is Only One Shelf}

Although there are few cases that there is only one shelf, it makes the foundation for multiple shelves case. Therefore we pick it up as a fundamental one. Suppose shelf position $k$ is from 1 to $L$ (Figure 2).

\begin{tabular}{|c}
\hline$k=L$ \\
\hline$\vdots$ \\
\hline$k=3$ \\
\hline$k=2$ \\
\hline$k=1$ \\
\hline
\end{tabular}

Figure 2. Shelf position

Suppose there are $N$ amount of goods $(i=1, \cdots, N)$. Set sales profit of goods $i$ as $H^{i}$. Table 1 shows the sales probabilities when each goods is placed at each shelf position. The values in this table are written for example. 
Table 1. Sales probability for each goods

\begin{tabular}{|c|c|c|c|c|c|c|c|c|c|c|c|}
\hline \multirow{3}{*}{$\begin{array}{l}\text { Day of the } \\
\text { Week }\end{array}$} & \multirow{3}{*}{ Time Zone $(t)$} & \multicolumn{3}{|c|}{ Shelf $j=1$} & \multicolumn{3}{|c|}{ Shelf $j=2$} & \multicolumn{4}{|c|}{ Shelf $j=m$} \\
\hline & & \multicolumn{3}{|c|}{ Shelf Position } & \multicolumn{3}{|c|}{ Shelf Position } & $\ldots$ & \multicolumn{3}{|c|}{ Shelf Position } \\
\hline & & $k=1$ & $\ldots$ & $k=L_{1}$ & $k=1$ & $\ldots$ & $k=L_{2}$ & $\ldots$ & $k=1$ & $\ldots$ & $k=L_{m}$ \\
\hline \multirow[t]{4}{*}{ (Mon.) } & $0-1(t=1)$ & 0.01 & $\ldots$ & & & & & & & & \\
\hline & $1-2(t=2)$ & 0.02 & & & & & & & & & \\
\hline & $\ldots$ & & & & & & & & & & \\
\hline & $23-24(t=24)$ & 0.03 & & & & & & & & & \\
\hline \multirow[t]{4}{*}{ (Tue.) } & $0-1(t=25)$ & 0.02 & & & & & & & & & \\
\hline & $1-2(t=26)$ & 0.02 & & & & & & & & & \\
\hline & $\ldots$ & & & & & & & & & & \\
\hline & $23-24 t=48()$ & 0.03 & & & & & & & & & \\
\hline$\ldots$ & $\ldots$ & $\ldots$ & $\ldots$ & $\ldots$ & $\ldots$ & $\ldots$ & $\ldots$ & $\ldots$ & $\ldots$ & $\ldots$ & $\ldots$ \\
\hline \multirow[t]{4}{*}{ (Sun.) } & $0-1(t=145)$ & 0.02 & & & & & & & & & \\
\hline & $1-2(t=146)$ & 0.03 & & & & & & & & & \\
\hline & $\ldots$ & & & & & & & & & & \\
\hline & $23-24(t=168)$ & 0.04 & & & & & & & & & \\
\hline
\end{tabular}

Suppose goods are sold in the period from $t_{1}$ to $t_{n}$. In addition, a new goods $i$ is replenished when goods $i$ is sold out.

Set the accumulated sales probability of goods $i$ in time zone $t$, shelf $j$, and shelf position $k$ in the table as $H K_{t, j, k}^{i}$.

Then, the sales probability $K_{t_{1} / t_{n}}^{i, j, k}$ of goods $i$ in the period will be described as follows.

$$
K_{t_{1} / t_{n}}^{i, j, k}=\sum_{t=1}^{n} H K_{t, k, j}^{i}
$$

This can take the value more than 1. For example, the value 2 means that 2 amount of goods were sold during the period. Set Benefit in the sales period from $t_{1}$ to $t_{n}$ as $P_{t_{1} / t_{n}}^{i, j, k}(i=1, \cdots, N)(j=1, \cdots, m)(k=1, \cdots, L)$ when goods $i$ is placed at shelf $j$ and shelf position $k$. Where Benefit means:

$$
\text { Benefit }=\text { SalesProbability } \times \text { SalesProfit }
$$

Therefore, this equation is represented as follows.

$$
P_{t_{1} / t_{n}}^{i, j, k}=K_{t_{1} / t_{n}}^{i, j, k} \cdot H^{i}
$$

where $j=1$ because one shelf case is considered here.

Set $x_{i, k}$ as:

$x_{i, k}=1$ : Goods $i$ is placed at shelf position $k$

$x_{i, k}=0:$ Else

Suppose only one goods can be placed at one shelf position and also suppose that goods is not placed for more than 2 shelf positions and is not placed in multiple shelves. Then constraints are described as follows.

$$
x_{i, k}=1,0(i=1, \cdots, N)(k=1, \cdots L)
$$




$$
\begin{aligned}
& \sum_{i=1}^{N} x_{i, k}=1(k=1, \cdots L) \\
& \sum_{k=1}^{N} x_{i, k} \leq 1(i=1, \cdots N)
\end{aligned}
$$

Under these constraints,

$$
\text { Maximize } J=\sum_{k=1}^{L} \sum_{i=1}^{N} P_{t_{1} / t_{n}}^{i, j, k} x_{i, k}
$$

\subsection{Case 2: The Case That There Are $m$ Shelves}

Suppose there are $m$ shelves (Figure 3). Set Benefit as $P_{t_{1} / t_{\eta}}^{i, j, k}(i=1, \cdots, N)(j=1, \cdots, m)\left(k=1, \cdots, L_{j}\right)$ where goods $i$ is placed at shelf position $k$ of shelf $j$. The sales period is the same with above stated (1).

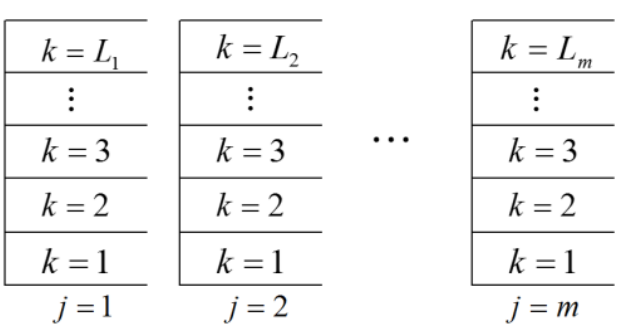

Figure 3. Shelf position under multiple shelves

Set $x_{i, j, k}$ as:

$x_{i, j, k}=1$ : Goods $i$ is placed at shelf position $k$ of shelf $j$

$x_{i, j, k}=0:$ Else

Suppose only one goods can be placed at one shelf position and also suppose that goods is not allowed to allocate in multiple shelf positions. Then constraints are described as follows. The sales period is the same with before.

$$
\begin{aligned}
& x_{i, j, k}=1,0(i=1, \cdots, N)(j=1, \cdots, m)\left(k=1, \cdots, L_{j}\right) \\
& \sum_{i=1}^{N} x_{i, j, k}=1(j=1, \cdots, m)\left(k=1, \cdots, L_{j}\right) \\
& \sum_{j=1}^{m} \sum_{k=1}^{L_{j}} x_{i, k, j} \leq 1(i=1, \cdots, N)
\end{aligned}
$$

Under these constraints,

$$
\text { Maximize } J=\sum_{i=1}^{N} \sum_{j=1}^{m} \sum_{k=1}^{L_{j}} P_{t_{1} / t_{n}}^{i, j, k} x_{i, j, k}
$$




\section{Algorithm}

We can make problem description as stated above, although these are somewhat under restricted cases. As far as only these are considered as they are, there is little difference between these and the conventional optimization problems. However, as soon as the number of involved shelves becomes larger, the number of variables dramatically grows greater, to which the application of Genetic Algorithm solution and Neural Network solutions may be appropriate. There are various means to solve this problem. When that variable takes the value of 0 or 1 , the application of genetic algorithm would be a good method. As is well known, the calculation volume reaches numerous or even infinite amounts in these problems when the number of variables increases. It is reported that GA is effective for these problems (Gen et al. (1995), Lin et al. (2005), Zhang et al. (2005)).

\section{A. The Variables}

Suppose the number of goods, shelf position, and shelf are 60, 2, 9 respectively. In this paper, shelf is expanded from 2 to 9 . Then the number of variables becomes 1080 .

$$
x_{i, j, k}=1,0(i=1, \cdots, 60),(j=1, \cdots, 9),(k=1,2)
$$

Therefore, set chromosome as follows.

$$
\begin{gathered}
X=\left(x_{1,1,1}, x_{2,1,1}, x_{3,1,1}, \cdots, x_{60,1,1}\right. \\
x_{1,1,2}, x_{2,1,2}, x_{3,1,2}, \cdots, x_{60,1,2}, \\
\vdots \\
x_{1,2,1}, x_{2,2,1}, x_{3,2,1}, \cdots, x_{60,2,1} \\
x_{1,2,2}, x_{2,2,2}, x_{3,2,2}, \cdots, x_{60,2,2} \\
\vdots \\
x_{1,9,1}, x_{2,9,1}, x_{3,9,1}, \cdots, x_{60,9,1} \\
\left.x_{1,9,2}, x_{2,9,2}, x_{3,9,2}, \cdots, x_{60,9,2}\right)
\end{gathered}
$$

\section{B. Initialize population}

Initialization of population is executed. The number of initial population is $M$. Here set $M=100$. Set gene at random and choose individual which satisfies constraints.

C. Selection

In this paper, we take elitism while selecting. Choose $P$ individuals in the order which take maximum score of objective function. Here, set $P=20$.

\section{Crossover}

Here, we take uniform crossover. Set crossover rate as:

$$
P_{c}=0.7
$$

E. Mutation

Set mutation rate as:

$$
P_{m}=0.01
$$

Algorithm of GA is exhibited at Table 2.

Table 2. Algorithm of multi-step tournament selection method

tep 1: Set maximum No. as $g_{\max }$, population size as $P$, crossover rate as $P_{c}$, mutation rate as $P_{m}$.

Step 2: Set $t=1$ for generation No. and generate initial solution matrix $x_{p}(t)=\left(x_{i k j}^{p}\right)(p=1, \cdots, M)$.

Step 3: Calculate Objective function $J\left(x_{p}(t)\right)$ for all solution matrix $x_{p}(t)(p=1, \cdots, P)$ in generation $t$.

Step 4: Set $t=t+1$ until $t>g_{\max }$.

Step 5: Crossover

Generate new individual by crossover utilizing the method of above stated $D$.

Step 6: Mutation 
Reproduce by mutation utilizing the method of above stated $E$.

Step 7: Calculate objective function for reproduction of generation $t$.

Step 8: Selection

Next generation is selected by elitism.

Go to Step 4.

Introducing the variable $y_{s}$ such that:

$$
y_{s}=i
$$

where

$$
s=k+(j-1) \cdot 2
$$

When

$$
x_{i, j, k}=1
$$

then (10) is expressed as:

$$
Y=\left(y_{1}, y_{2}, \cdots, y_{18}\right)
$$

\section{Numerical Example}

Now, we execute numerical example using POS sales data. Numerical example is executed in "Case 2" of 2 (2).

\begin{tabular}{|c|c|c|}
\hline Lot $i$ & Sales Price & \\
\hline 1 & & \multirow{9}{*}{ For Women } \\
\hline 2 & & \\
\hline 3 & & \\
\hline 4 & & \\
\hline 5 & & \\
\hline 6 & & \\
\hline 7 & & \\
\hline 8 & & \\
\hline 9 & & \\
\hline 10 & & \multirow{8}{*}{ For Men } \\
\hline 11 & & \\
\hline 12 & & \\
\hline 13 & & \\
\hline 14 & & \\
\hline 15 & & \\
\hline 16 & & \\
\hline 17 & & \\
\hline 18 & & \multirow{3}{*}{ For Kids } \\
\hline 19 & & \\
\hline 20 & & \\
\hline
\end{tabular}
Suppose the sales period is 5 days for Monday through Saturday. Table 3 shows the unit sales profit $H^{i}$ of each goods.

Table 3. Unit sales price and sales profit of each goods

Supposing a general daytime retail store, we set opening time to be 9 through 18 o'clock. Table 4 shows the sales probabilities of lot $i$ as an example. 
Table 5 shows the sales probability by shelf for each shelf position. Table 6 shows the value in which Table 4 and Table 5 are multiplied. Table 7 shows the benefit Table in which accumulated probability of Table 6 and Sales Profit of Table 3 are multiplied.

Table 4. Sales probability of lot $i$ (time zone)

\begin{tabular}{|c|c|c|c|c|c|}
\hline Day of the Week & Time Zone $(t)$ & Sales Probability & Day of the Week & Time Zone $(t)$ & Sales Probability \\
\hline \multirow[t]{9}{*}{ (Mon.) } & $9-10$ & & \multirow[t]{9}{*}{ (Thu.) } & $9-10$ & \\
\hline & $10-11$ & & & $10-11$ & \\
\hline & $11-12$ & & & $11-12$ & \\
\hline & $12-13$ & & & $12-13$ & \\
\hline & $13-14$ & & & $13-14$ & \\
\hline & $14-15$ & & & $14-15$ & \\
\hline & $15-16$ & & & $15-16$ & \\
\hline & $16-17$ & & & $16-17$ & \\
\hline & $17-18$ & & & $17-18$ & \\
\hline \multirow[t]{9}{*}{ (Tue.) } & $9-10$ & & \multirow[t]{9}{*}{ (Fri.) } & $9-10$ & \\
\hline & $10-11$ & & & $10-11$ & \\
\hline & $11-12$ & & & $11-12$ & \\
\hline & $12-13$ & & & $12-13$ & \\
\hline & $13-14$ & & & 13-14 & \\
\hline & $14-15$ & & & $14-15$ & \\
\hline & $15-16$ & & & $15-16$ & \\
\hline & $16-17$ & & & $16-17$ & \\
\hline & 17-18 & & & 17-18 & \\
\hline \multirow[t]{9}{*}{ (Wed.) } & $9-10$ & & \multirow[t]{9}{*}{ (Sat.) } & $9-10$ & \\
\hline & $10-11$ & & & $10-11$ & \\
\hline & $11-12$ & & & $11-12$ & \\
\hline & $12-13$ & & & 12-13 & \\
\hline & $13-14$ & & & $13-14$ & \\
\hline & 14-15 & & & 14-15 & \\
\hline & $15-16$ & & & $15-16$ & \\
\hline & 16-17 & & & 16-17 & \\
\hline & $17-18$ & & & $17-18$ & \\
\hline
\end{tabular}

Table 5. Sales probability of lot $i$ (shelf position)

\begin{tabular}{|c|c|c|c|c|c|c|c|c|c|c|c|}
\hline \multirow{2}{*}{\multicolumn{2}{|c|}{$\begin{array}{l}\text { Shelf } j=1 \\
\text { Shelf Position }\end{array}$}} & \multirow{2}{*}{\multicolumn{2}{|c|}{$\begin{array}{l}\text { Shelf } j=2 \\
\text { Shelf Position }\end{array}$}} & \multicolumn{2}{|c|}{ Shelf $j=3$} & \multicolumn{2}{|c|}{ Shelf $j=4$} & \multicolumn{2}{|c|}{ Shelf $j=5$} & \multicolumn{2}{|c|}{ Shelf $j=6$} \\
\hline & & & & \multicolumn{2}{|c|}{ Shelf Position } & \multicolumn{2}{|c|}{ Shelf Position } & \multicolumn{2}{|c|}{ Shelf Position } & \multicolumn{2}{|c|}{ Shelf Position } \\
\hline$k=1$ & $k=2$ & $k=1$ & $k=2$ & $k=1$ & $k=2$ & $k=1$ & $k=2$ & $k=1$ & $k=2$ & $k=1$ & $k=2$ \\
\hline \multirow[t]{5}{*}{1.0} & 1.0 & 1.0 & 1.0 & 1.0 & 1.0 & 1.1 & 1.1 & 1.2 & 1.2 & 1.2 & 1.2 \\
\hline & & & Shelf & $=7$ & Shelf & $=8$ & Shelf & $=9$ & & & \\
\hline & & & Shelf & osition & Shelf & osition & Shelf I & osition & & & \\
\hline & & & $k=1$ & $k=2$ & $k=1$ & $k=2$ & $k=1$ & $k=2$ & & & \\
\hline & & & 1.1 & 1.1 & 1.0 & 1.0 & 0.9 & 0.9 & & & \\
\hline
\end{tabular}


In Table 5, shelf $j=5$ is located near the entrance therefore the table value reflects this condition.

Table 6. Sales probability of lot $i$

\begin{tabular}{|c|c|c|c|c|c|c|c|c|c|c|c|c|}
\hline \multirow{3}{*}{$\begin{array}{l}\text { Day of } \\
\text { the Week }\end{array}$} & \multirow{3}{*}{ Time Zone $(t)$} & \multicolumn{11}{|c|}{ Sales Probability } \\
\hline & & \multicolumn{2}{|c|}{ Shelf $j=1$} & \multicolumn{2}{|c|}{$\begin{array}{l}\text { Shelf } \\
j=2\end{array}$} & \multicolumn{2}{|c|}{$\begin{array}{l}\text { Shelf } \\
j=3\end{array}$} & \multirow{2}{*}{$\begin{array}{l}\cdots \\
\cdots\end{array}$} & \multicolumn{2}{|c|}{$\begin{array}{l}\text { Shelf } \\
j=8\end{array}$} & \multicolumn{2}{|c|}{$\begin{array}{l}\text { Shelf } \\
j=9\end{array}$} \\
\hline & & $k=1$ & $k=2$ & $k=1$ & $k=2$ & $k=1$ & $k=2$ & & $k=1$ & $k=2$ & $k=1$ & $k=2$ \\
\hline \multirow[t]{9}{*}{ (Mon.) } & $9-10$ & & & & & & & $\ldots$. & & & & \\
\hline & $10-11$ & & & & & & & $\ldots$ & & & & \\
\hline & $11-12$ & & & & & & & $\ldots$ & & & & \\
\hline & $12-13$ & & & & & & & $\ldots$. & & & & \\
\hline & $13-14$ & & & & & & & $\ldots$ & & & & \\
\hline & $14-15$ & & & & & & & $\ldots$ & & & & \\
\hline & $15-16$ & & & & & & & $\ldots$ & & & & \\
\hline & $16-17$ & & & & & & & $\ldots$ & & & & \\
\hline & $17-18$ & & & & & & & $\ldots$ & & & & \\
\hline \multirow[t]{9}{*}{ (Tue.) } & $9-10$ & & & & & & & $\ldots$ & & & & \\
\hline & $10-11$ & & & & & & & $\ldots$ & & & & \\
\hline & $11-12$ & & & & & & & $\ldots$ & & & & \\
\hline & $12-13$ & & & & & & & $\ldots$ & & & & \\
\hline & $13-14$ & & & & & & & $\ldots$ & & & & \\
\hline & $14-15$ & & & & & & & $\ldots$ & & & & \\
\hline & $15-16$ & & & & & & & $\ldots$ & & & & \\
\hline & $16-17$ & & & & & & & $\ldots$ & & & & \\
\hline & $17-18$ & & & & & & & $\ldots$ & & & & \\
\hline & & & & & $\vdots$ & & & & & & & \\
\hline \multirow[t]{9}{*}{ (Sat.) } & $9-10$ & & & & & & & $\ldots$ & & & & \\
\hline & $10-11$ & & & & & & & $\ldots$ & & & & \\
\hline & $11-12$ & & & & & & & $\ldots$ & & & & \\
\hline & $12-13$ & & & & & & & $\ldots$ & & & & \\
\hline & $13-14$ & & & & & & & $\ldots$ & & & & \\
\hline & $14-15$ & & & & & & & $\ldots$ & & & & \\
\hline & $15-16$ & & & & & & & $\ldots$ & & & & \\
\hline & $16-17$ & & & & & & & $\ldots$ & & & & \\
\hline & $17-18$ & & & & & & & $\ldots$ & & & & \\
\hline
\end{tabular}

Table 7 shows the benefit when each goods is placed at each shelf position of each shelf.

Table 7. Benefit table

\begin{tabular}{lllllllllll}
\hline & \multicolumn{2}{l}{ Shelf 1 } & \multicolumn{2}{l}{ Shelf 2 } & \multicolumn{2}{l}{ Shelf 3 } & \multicolumn{2}{l}{ Shelf 4 } & \multicolumn{2}{l}{ Shelf 5 } \\
\cline { 2 - 13 } & Shelf Position & \multicolumn{2}{l}{ Shelf Position } & \multicolumn{2}{l}{ Shelf Position } & \multicolumn{2}{l}{ Shelf Position } & \multicolumn{2}{l}{ Shelf Position } \\
\hline Lot $i$ & 1 & 2 & 1 & 2 & 1 & 2 & 1 & 2 & 1 & 2 \\
\hline 1 & 500 & 800 & 510 & 810 & 510 & 810 & 630 & 1270 & 550 & 1010 \\
\hline 2 & 540 & 924 & 520 & 900 & 450 & 850 & 450 & 850 & 450 & 850 \\
\hline 3 & 820 & 390 & 820 & 390 & 820 & 390 & 912 & 500 & 800 & 360 \\
\hline 4 & 400 & 841 & 400 & 841 & 400 & 841 & 419 & 859 & 400 & 841 \\
\hline
\end{tabular}




\begin{tabular}{|c|c|c|c|c|c|c|c|c|c|c|}
\hline 5 & 836 & 401 & 820 & 390 & 820 & 390 & 820 & 390 & 800 & 360 \\
\hline 6 & 410 & 840 & 410 & 840 & 420 & 860 & 410 & 840 & 410 & 840 \\
\hline 7 & 820 & 390 & 835 & 400 & 820 & 390 & 820 & 390 & 800 & 380 \\
\hline 8 & 670 & 290 & 670 & 290 & 670 & 290 & 670 & 290 & 670 & 290 \\
\hline 9 & 820 & 390 & 820 & 390 & 913 & 501 & 820 & 390 & 790 & 350 \\
\hline 10 & 500 & 900 & 500 & 900 & 400 & 800 & 400 & 800 & 500 & 1000 \\
\hline 11 & 500 & 900 & 539 & 923 & 450 & 850 & 450 & 850 & 450 & 850 \\
\hline 12 & 560 & 900 & 560 & 900 & 400 & 850 & 400 & 850 & 630 & 1140 \\
\hline 13 & 670 & 290 & 670 & 290 & 670 & 290 & 670 & 290 & 670 & 290 \\
\hline 14 & 800 & 380 & 800 & 380 & 800 & 380 & 800 & 380 & 815 & 403 \\
\hline 15 & 821 & 395 & 821 & 395 & 822 & 396 & 822 & 396 & 801 & 401 \\
\hline 16 & 370 & 840 & 370 & 840 & 370 & 840 & 400 & 900 & 400 & 900 \\
\hline 17 & 500 & 800 & 520 & 800 & 520 & 800 & 520 & 800 & 610 & 1100 \\
\hline 18 & 680 & 295 & 680 & 295 & 680 & 295 & 680 & 295 & 680 & 295 \\
\hline 19 & 790 & 310 & 790 & 310 & 790 & 310 & 790 & 310 & 790 & 310 \\
\hline 20 & 390 & 900 & 390 & 900 & 380 & 830 & 460 & 990 & 460 & 990 \\
\hline 21 & 475 & 767 & 478 & 783 & 483 & 777 & 600 & 1244 & 522 & 976 \\
\hline 22 & 513 & 895 & 494 & 868 & 424 & 823 & 422 & 822 & 422 & 820 \\
\hline 23 & 789 & 361 & 785 & 356 & 786 & 362 & 884 & 470 & 769 & 333 \\
\hline 24 & 372 & 806 & 372 & 808 & 366 & 810 & 386 & 831 & 366 & 809 \\
\hline 25 & 804 & 368 & 786 & 361 & 794 & 361 & 793 & 362 & 769 & 332 \\
\hline 26 & 376 & 813 & 384 & 815 & 391 & 828 & 384 & 812 & 378 & 810 \\
\hline 27 & 788 & 359 & 802 & 365 & 790 & 362 & 788 & 360 & 770 & 346 \\
\hline 28 & 635 & 257 & 643 & 263 & 640 & 258 & 638 & 263 & 636 & 260 \\
\hline 29 & 788 & 361 & 795 & 361 & 880 & 475 & 788 & 358 & 765 & 325 \\
\hline 30 & 469 & 867 & 468 & 873 & 367 & 766 & 366 & 767 & 471 & 970 \\
\hline 31 & 465 & 875 & 509 & 888 & 423 & 823 & 423 & 824 & 424 & 819 \\
\hline 32 & 529 & 867 & 528 & 868 & 369 & 823 & 366 & 823 & 602 & 1114 \\
\hline 33 & 642 & 265 & 643 & 264 & 642 & 264 & 640 & 262 & 641 & 259 \\
\hline 34 & 771 & 352 & 775 & 350 & 774 & 354 & 768 & 345 & 781 & 368 \\
\hline 35 & 786 & 361 & 787 & 362 & 792 & 367 & 795 & 369 & 774 & 370 \\
\hline 36 & 339 & 815 & 340 & 815 & 335 & 813 & 368 & 871 & 374 & 868 \\
\hline 37 & 466 & 770 & 488 & 775 & 491 & 770 & 490 & 775 & 579 & 1073 \\
\hline 38 & 653 & 269 & 651 & 265 & 653 & 264 & 648 & 265 & 654 & 262 \\
\hline 39 & 755 & 284 & 758 & 275 & 761 & 283 & 762 & 285 & 764 & 284 \\
\hline 40 & 359 & 874 & 360 & 874 & 347 & 796 & 434 & 957 & 432 & 957 \\
\hline 41 & 436 & 736 & 445 & 754 & 454 & 748 & 565 & 1213 & 492 & 948 \\
\hline 42 & 479 & 866 & 464 & 840 & 391 & 785 & 390 & 788 & 393 & 787 \\
\hline 43 & 765 & 330 & 756 & 334 & 763 & 325 & 851 & 437 & 737 & 304 \\
\hline 44 & 337 & 780 & 338 & 781 & 342 & 779 & 361 & 801 & 341 & 780 \\
\hline 45 & 774 & 343 & 756 & 335 & 755 & 330 & 757 & 332 & 735 & 301 \\
\hline 46 & 347 & 782 & 348 & 782 & 358 & 804 & 349 & 783 & 347 & 782 \\
\hline 47 & 764 & 329 & 772 & 338 & 755 & 335 & 758 & 331 & 744 & 323 \\
\hline 48 & 611 & 233 & 614 & 226 & 609 & 227 & 605 & 232 & 605 & 228 \\
\hline 49 & 760 & 334 & 760 & 328 & 848 & 441 & 759 & 330 & 726 & 294 \\
\hline
\end{tabular}




\begin{tabular}{lllllllllll}
\hline 50 & 442 & 840 & 445 & 838 & 338 & 736 & 344 & 735 & 444 & 940 \\
\hline 51 & 437 & 840 & 482 & 868 & 391 & 794 & 392 & 788 & 387 & 792 \\
\hline 52 & 500 & 836 & 505 & 839 & 340 & 787 & 338 & 785 & 568 & 1079 \\
\hline 53 & 612 & 230 & 606 & 227 & 605 & 230 & 612 & 232 & 612 & 226 \\
\hline 54 & 740 & 319 & 744 & 315 & 739 & 322 & 742 & 319 & 760 & 340 \\
\hline 55 & 765 & 332 & 756 & 332 & 761 & 337 & 763 & 337 & 744 & 343 \\
\hline 56 & 307 & 776 & 307 & 784 & 308 & 783 & 342 & 840 & 337 & 842 \\
\hline 57 & 436 & 743 & 465 & 739 & 460 & 744 & 455 & 736 & 553 & 1038 \\
\hline 58 & 616 & 231 & 619 & 238 & 620 & 231 & 624 & 239 & 617 & 235 \\
\hline 59 & 727 & 254 & 729 & 249 & 735 & 247 & 734 & 253 & 734 & 245 \\
\hline 60 & 335 & 839 & 326 & 843 & 316 & 769 & 403 & 926 & 405 & 926 \\
\hline
\end{tabular}

\begin{tabular}{lllllllll}
\hline & \multicolumn{3}{l}{ Shelf 6} & \multicolumn{2}{l}{ Shelf 7} & \multicolumn{2}{l}{ Shelf 8} & \multicolumn{2}{l}{ Shelf 9} \\
\cline { 2 - 10 } & Shelf Position & \multicolumn{2}{l}{ Shelf Position } & \multicolumn{2}{l}{ Shelf Position } & \multicolumn{2}{l}{ Shelf Position } \\
\hline Lot $i$ & 1 & 2 & 1 & 2 & 1 & 2 & 1 & 2 \\
\hline 1 & 540 & 1010 & 550 & 1100 & 560 & 1100 & 560 & 1010 \\
\hline 2 & 450 & 850 & 450 & 850 & 450 & 850 & 450 & 850 \\
\hline 3 & 800 & 360 & 670 & 300 & 670 & 300 & 670 & 300 \\
\hline 4 & 400 & 841 & 400 & 841 & 400 & 841 & 400 & 841 \\
\hline 5 & 800 & 360 & 670 & 300 & 670 & 300 & 670 & 300 \\
\hline 6 & 410 & 840 & 410 & 840 & 410 & 840 & 410 & 840 \\
\hline 7 & 800 & 380 & 670 & 310 & 670 & 310 & 670 & 310 \\
\hline 8 & 670 & 290 & 680 & 300 & 680 & 300 & 689 & 301 \\
\hline 9 & 790 & 350 & 660 & 280 & 660 & 280 & 660 & 280 \\
\hline 10 & 500 & 1000 & 500 & 1000 & 614 & 1272 & 500 & 1000 \\
\hline 11 & 450 & 850 & 450 & 850 & 450 & 850 & 450 & 850 \\
\hline 12 & 550 & 1000 & 550 & 1000 & 550 & 1000 & 550 & 1000 \\
\hline 13 & 670 & 290 & 688 & 300 & 690 & 302 & 688 & 300 \\
\hline 14 & 800 & 380 & 680 & 300 & 680 & 300 & 680 & 300 \\
\hline 15 & 801 & 400 & 681 & 295 & 681 & 296 & 911 & 500 \\
\hline 16 & 629 & 1139 & 460 & 990 & 460 & 990 & 460 & 990 \\
\hline 17 & 615 & 1100 & 615 & 1273 & 600 & 1200 & 600 & 1100 \\
\hline 18 & 680 & 295 & 691 & 303 & 685 & 300 & 685 & 300 \\
\hline 19 & 814 & 402 & 680 & 290 & 680 & 290 & 680 & 290 \\
\hline 20 & 460 & 990 & 460 & 990 & 460 & 990 & 605 & 1191 \\
\hline 21 & 505 & 979 & 525 & 1074 & 531 & 1071 & 527 & 982 \\
\hline 22 & 416 & 822 & 418 & 817 & 424 & 817 & 421 & 818 \\
\hline 23 & 771 & 329 & 635 & 274 & 638 & 265 & 638 & 275 \\
\hline 24 & 366 & 811 & 371 & 814 & 372 & 808 & 365 & 810 \\
\hline 25 & 771 & 332 & 636 & 273 & 637 & 266 & 642 & 271 \\
\hline 26 & 379 & 814 & 383 & 808 & 375 & 810 & 378 & 809 \\
\hline 27 & 770 & 355 & 637 & 282 & 643 & 280 & 636 & 281 \\
\hline 28 & 637 & 262 & 645 & 274 & 647 & 268 & 657 & 269 \\
\hline 30 & 763 & 319 & 628 & 252 & 629 & 253 & 633 & 251 \\
\hline 31 & 465 & 973 & 468 & 974 & 587 & 1238 & 475 & 966 \\
\hline & & & & & & & & 815 \\
\hline
\end{tabular}




\begin{tabular}{|c|c|c|c|c|c|c|c|c|}
\hline 32 & 517 & 974 & 521 & 965 & 519 & 965 & 523 & 966 \\
\hline 33 & 638 & 262 & 654 & 274 & 665 & 277 & 662 & 266 \\
\hline 34 & 766 & 354 & 654 & 265 & 653 & 275 & 654 & 272 \\
\hline 35 & 767 & 366 & 652 & 264 & 656 & 264 & 879 & 465 \\
\hline 36 & 598 & 1107 & 434 & 964 & 427 & 965 & 427 & 964 \\
\hline 37 & 589 & 1072 & 581 & 1246 & 567 & 1175 & 575 & 1074 \\
\hline 38 & 646 & 265 & 661 & 276 & 656 & 272 & 660 & 269 \\
\hline 39 & 779 & 377 & 653 & 260 & 651 & 261 & 651 & 259 \\
\hline 40 & 429 & 962 & 430 & 959 & 425 & 962 & 575 & 1157 \\
\hline 41 & 479 & 946 & 490 & 1044 & 499 & 1036 & 504 & 949 \\
\hline 42 & 385 & 787 & 391 & 789 & 391 & 793 & 395 & 789 \\
\hline 43 & 738 & 303 & 612 & 241 & 612 & 244 & 605 & 244 \\
\hline 44 & 342 & 784 & 335 & 786 & 343 & 785 & 342 & 780 \\
\hline 45 & 738 & 304 & 615 & 242 & 605 & 236 & 610 & 243 \\
\hline 46 & 350 & 785 & 347 & 775 & 351 & 781 & 352 & 778 \\
\hline 47 & 743 & 315 & 614 & 252 & 615 & 249 & 606 & 246 \\
\hline 48 & 613 & 231 & 621 & 244 & 625 & 243 & 633 & 237 \\
\hline 49 & 732 & 295 & 601 & 224 & 600 & 220 & 595 & 217 \\
\hline 50 & 442 & 939 & 435 & 937 & 549 & 1217 & 439 & 942 \\
\hline 51 & 387 & 788 & 386 & 793 & 390 & 795 & 394 & 785 \\
\hline 52 & 492 & 936 & 492 & 943 & 485 & 945 & 490 & 939 \\
\hline 53 & 607 & 235 & 629 & 241 & 629 & 240 & 628 & 240 \\
\hline 54 & 740 & 320 & 621 & 240 & 615 & 245 & 617 & 239 \\
\hline 55 & 745 & 336 & 622 & 230 & 618 & 235 & 856 & 435 \\
\hline 56 & 573 & 1081 & 396 & 935 & 398 & 931 & 395 & 926 \\
\hline 57 & 550 & 1039 & 556 & 1217 & 543 & 1143 & 538 & 1045 \\
\hline 58 & 618 & 236 & 627 & 244 & 629 & 240 & 630 & 243 \\
\hline 59 & 751 & 340 & 615 & 233 & 622 & 233 & 621 & 228 \\
\hline 60 & 395 & 930 & 395 & 934 & 399 & 926 & 550 & 1129 \\
\hline
\end{tabular}

Experimental results are as follows. The expression Eq. (10) is complicated. Therefore, we use expression by Eq. (15). A sample set of initial population is exhibited in Table 8.

Table 8. A sample set of initial population

\begin{tabular}{|c|c|c|c|c|c|c|c|c|c|c|c|c|c|c|c|c|c|c|c|c|}
\hline$Y_{1}=$ & 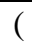 & 50 & 26 , & 16 , & 49 , & 13 , & 12 , & 43 , & 8, & 40 , & 36 , & 21 , & 53 , & 35 , & 60 , & 7, & 31 , & 39 , & 22 & ) \\
\hline$Y_{2}=$ & ( & 15 , & 30 , & 22 , & 3 , & 32 , & 9 , & 5 , & 8, & 55 & 27 , & 13 , & 1, & 45 , & 49 , & 43 , & 53 , & 14 , & 58 & ) \\
\hline \multirow[t]{2}{*}{$Y_{3}=$} & ( & 1 , & 13 , & 3 , & 42 , & 2 , & 33 , & 15 , & 21 , & 36 & 45 , & 20 , & 5 , & 58 , & 48 , & 50 , & 18 , & 43 , & 32 & ) \\
\hline & & & & & & & & & & & $\vdots$ & & & & & & & & & \\
\hline$Y_{98}=$ & ( & 37 , & 58, & 60 , & 7 & 47 , & 44, & 56 , & 27, & 29 , & 50 , & 53 , & 4, & 31 , & 21 , & 38 , & 9, & 25 , & 23 & ) \\
\hline$Y_{99}=$ & ( & 33 , & 31 , & 2, & 21 , & 18 , & 25 , & 8 , & 56 , & 4, & 48 , & 13 , & 17 , & 30 , & 14 & 54 & 26 , & 5 , & 15 & ) \\
\hline$Y_{100}=$ & ( & 15 , & 13 , & 11 , & 32 , & 53 , & 10 , & 57, & 35 , & 55 , & 42 , & 37 , & 1 , & 36 , & 23 , & 31 , & 56 , & 21 , & 14 & ) \\
\hline
\end{tabular}

Convergence process is exhibited in Figure 4. 


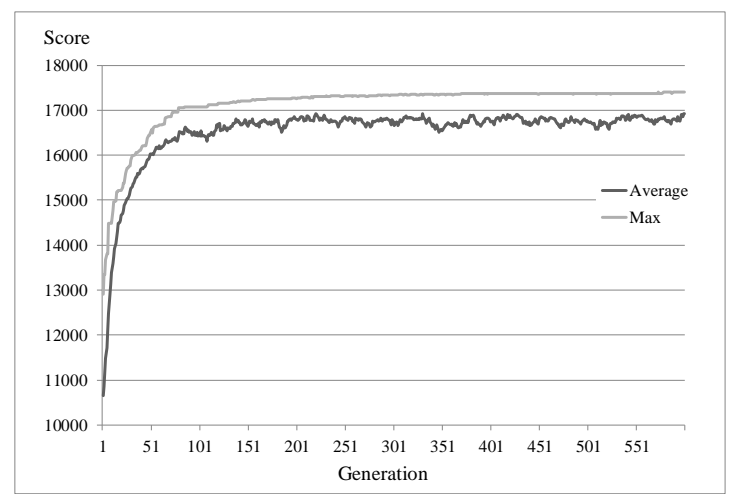

Figure 4. Convergence process of Case 2

The problem is simple, so combination of genotype for crossover saturates in the $578^{\text {th }}$ generation. Genotype in which objective function becomes maximum is as follows.

$$
Y=(5,2,7,11,9,6,3,1,14,12,19,16,18,17,13,10,15,20)
$$

This coincides with the result of optimal solution by the calculation of all considerable cases, therefore it coincides with a theoretical optimal solution. We take up simple problem and we can confirm the effectiveness of GA approach. Further study for complex problems should be examined hereafter.

\section{Remarks}

As there are few papers made on this theme, we constructed prototype version before (Takeyasu et al.,2008). In this paper, we examined the problem that did not allow goods to be allocated in multiple shelves and introduced the concept of sales profits and sales probabilities. An application to the shop with POS sales data was executed. We can see that genetic algorithm is effective for this problem.

In practice, following themes occur.

1. Sales probabilities should be arranged correctly.

2. There are various types of shelves corresponding to goods characteristics (For example, cold storage goods).

3. Furthermore, genotype must be devised in construction when there are huge number of goods and shelves.

For these issues, expanded version of the paper will be built hereafter consecutively. As for 1, constraints are relaxed than those of this paper. As for 2, expansion is easy to make. As for 3, constructing genotype from the shelf side would bear much more simple expression.

We have examined various patterns on this problem type where the number of shelf position and those of shelf are different. They also include the different pattern that "Goods are allowed to allocate in multiple shelves" and "Goods are not allowed to allocate in multiple shelves".

These are arranged by the viewpoint of variable number (The number of shelf position and those of shelf are involved), lot number, presence of the permission of allocating goods in multiple shelves. Then the convergence number will be different accordingly.

We summarize here and compare them. There are 9 papers. Comparison is exhibited in Table 9.

In the table, the notation means:

32: Number of convergence

(20): Lot Number

[15]: Reference Number

Table 9. Comparison with other cases

\begin{tabular}{llllll}
\hline Variables & 12 & 15 & 16 & 18 & 20 \\
\hline Goods are allowed to allocate in multiple shelves & 63 & 170 & 489 & 125 & \\
\hline
\end{tabular}




\begin{tabular}{llllll}
\hline & $(20)$ & $(20)$ & $(40)$ & $(20)$ & \\
& {$[6]$} & {$[4]$} & {$[7]$} & {$[5]$} & \\
& & & & 184 & \\
& & & & $(25)$ & \\
& & & & {$[2]$} & \\
\hline Goods are not allowed to allocate in multiple shelves & 408 & & 321 & 43 \\
& & $(60)$ & $(40)$ & $(20)$ \\
& & {$[8]$} & {$[3]$} & {$[1]$}
\end{tabular}

Note. number of references in Table 9 are as follows:

[1] Takeyasu, K., Higuchi, Y. (2011). Optimization in Allocating Goods to Shop Shelves, International Journal of Information Systems for Logistics and Management, Vol.6, No.2, pp.1-6.

[2] Takeyasu, K., Higuchi, Y. (2013). Optimization in Allocating Goods to Shop Shelves Utilizing Genetic Algorithm - An Application to the Shop Shelves for Yogurt Sales data-, Journal of Communication and Computer, Vol. 10, No.3, pp.416-424.

[3] Takeyasu, K., Higuchi, Y. (2013). Optimization in Allocating Goods to Shop Shelves Utilizing Genetic Algorithm for Yogurt Sales data, Chinese Business ReviewUSA-China Business Review, Vol.12, No.7, pp.497-508.

[4] Shitara, A., Higuchi, Y., Takeyasu, D., Takeyasu, K. (2015). Optimization in Allocating Goods to Shop Shelves Utilizing Genetic Algorithm Under the Introduction of Sales Probabilities, Journal of Communication and Computer, Vol12, No.4, pp.155-163.

[5] Suzuki, K., Higuchi, Y., Takeyasu, K. (2015). Optimization in Allocating Goods to Shop Shelves for Cup Noodles, Journal of Computations \& Modelling, Vol.5, No.4, pp.1-25.

[6] Higuchi, K., Takeyasu, K. (2016). Optimization in Allocating Goods to Shop Shelves Utilizing Genetic Algorithm under Expanded Shelf Position Case, Journal of Computations \& Modelling, Vol.6, No.2, pp.15-31.

[7] Takeyasu, K., Higuchi, Y. (2016). Utilization of Genetic Algorithm in Allocating Goods to Shop Shelves, Business and Management Research, Vol.5. No.4, pp.1-13.

[8] Higuchi, K., Takeyasu, K. (2017). Utilization of Genetic Algorithm in Allocating Goods to Shop Shelves Under the Introduction of Sales Probabilities, Bulletin of Kagawa Junior College, Vol.45, pp.29-41.

We can observe the following points.

$>$ Generally, the convergence number is greater in the case "Goods are not allowed to allocate in multiple shelves" than the case "Goods are allowed to allocate in multiple shelves".

$>$ Generally, the convergence number is greater for the case of greater lot number.

If the variable number is greater such that 200, 2000 or 20000, then the convergence number would be greater. Such confirmation is our future works to be investigated.

\section{Conclusion}

How to allocate goods in shop shelves makes great influence to sales amount. Searching best fit allocation of goods to shelves is a kind of combinatorial problem. This becomes a problem of integer programming and utilizing genetic algorithm may be an effective method. Reviewing past researches, there were few researches made on this. Formerly, we had presented papers concerning optimization in allocating goods to shop shelves utilizing genetic algorithm. In those papers, the problem that goods were not allowed to allocate in multiple shelves and the problem that goods were allowed to allocate in multiple shelves were pursued. In this paper, we examined the problem that did not allow goods to be allocated in multiple shelves and introduced the concept of sales profits and sales probabilities. Expansion of shelf was executed. Optimization in allocating goods to shop shelves was investigated. An application to the convenience store with POS sales data of cup noodles was executed. Utilizing genetic algorithm, optimum solution was pursued and verified by a numerical example.

Comparison with other past papers was executed. We could observe the following points.

$>$ Generally, the convergence number is greater in the case "Goods are not allowed to allocate in multiple shelves" 
than the case "Goods are allowed to allocate in multiple shelves".

$>$ Generally, the convergence number is greater for the case of greater lot number.

If the variable number is greater such that 200,2000 or 20000 , then the convergence number would be greater. Such confirmation is our future works to be investigated.

Various patterns of problems should be examined hereafter.

\section{References}

Arial, M., \& Masui, Y. (2006). Optimization of maritime container-transportation network through the use of genetic algorithm. Japan Ship-Marine Engineering Society, 4, 55-61.

Atsumi, S. (2008). Shop Lay Out (in Japanese). Jitsumu-Kyoiku-Press.

Gen, M., \& Cheng, R. (1997). Genetic Algorithms \& Engineering Design. John Wiley \& Sons, Inc.

Gen, M., Ida, K., \& Li, Y. (1995). Solving multi objective solid transportation problem by genetic algorithm. JIMA, 46(5), 445-454.

Higuchi, K., \& Takeyasu, K. (2016). Optimization in Allocating Goods to Shop Shelves Utilizing Genetic Algorithm under Expanded Shelf Position Case. Journal of Computations \& Modelling, 6(2), 15-31.

Higuchi, K., \& Takeyasu, K. (2017). Utilization of Genetic Algorithm in Allocating Goods to Shop Shelves Under the Introduction of Sales Probabilities. Bulletin of Kagawa Junior College, 45, 29-41.

Ito, T., \& Irohara, T. (2006). Multi-floor Facility Layout Technique Including Determination of Detailed $I / O$ Location. JIMA, 57(5), 395-403.

Kohno, H. (2006). Making Charming Shop (in Japanese). Paru-Press.

Korte, B., \& Vygeu, J. (2005). Combinatorial Optimization. Springer.

Michalewicz, Z. (1999) .Genetic Algorithms + Data Structures = Evolution Programs. Springer.

Nagashima, Y. (2005). Shop Arrangement and Displaying (in Japanese). Nihon-Jitsugyo-Press.

Shitara, A., Higuchi, Y., Takeyasu, D., \& Takeyasu, K. (2015). Optimization in Allocating Goods to Shop Shelves Utilizing Genetic Algorithm Under the Introduction of Sales Probabilities. Journal of Communication and Computer, 12(4), 155-163.

Suzuki, K., Higuchi, Y., \& Takeyasu, K. (2015). Optimization in Allocating Goods to Shop Shelves for Cup Noodles. Journal of Computations \& Modelling, 5(4), 1-25.

Takeyasu, K., \& Higuchi, Y. (2011). Optimization in Allocating Goods to Shop Shelves. International Journal of Information Systems for Logistics and Management, 6(2), 1-6.

Takeyasu, K., \& Higuchi, Y. (2013). Optimization in Allocating Goods to Shop Shelves Utilizing Genetic Algorithm - An Application to the Shop Shelves for Yogurt Sales data. Journal of Communication and Computer, 10(3), 416-424.

Takeyasu, K., \& Higuchi, Y. (2013). Optimization in Allocating Goods to Shop Shelves Utilizing Genetic Algorithm for Yogurt Sales data. Chinese Business Review, 12(7), 497-508.

Takeyasu, K., \& Higuchi, Y. (2016). Utilization of Genetic Algorithm in Allocating Goods to Shop Shelves. Business and Management Research, 5(4), 1-13.

Toyama, H., Ida, K., \& Teramatsu, C. (2006). A proposal of a genetic algorithm for fixed-charge transportation problem and related numerical experiments. JIMA, 57(3), 227-230.

Wu, Y., \& Appleton, E. (2002). The optimization of block layout and aisle structure by a genetic algorithm. Computers \& Industrial Engineering, 41, 371-382.

Yamada, T., \& Irohara, T. (2006). Detailed Layout Design Methodology using Mixed Integer Programming and Simulated Annealing. JIMA, 57(1), 39-50.

Yamada, T., Irohara, T., \& Fujikawa, H. (2004). Design methodology for the Facility Layout Problem to Consider the Aisle Structure and Intra-Department Material Flow. JIMA, 55(3), 111-120. 